\title{
A SURVEY ON DELAY TOLERANT NETWORK
}

\begin{tabular}{|l|l|l|}
\hline Navyashree.TM, (M.Tech) & Shashikala, (Ph.D) & Dr.Kavitha.C, Ph.D \\
B.N.M.Institute of Technology, & Associate professor & Professor \\
Bangalore, Karnataka, India. & B.N.M.Institute of Technology, & B.N.M.Institute of Technology, \\
navyashree.tm@gmail.com & Bangalore, Karnataka, India. & Bangalore, Karnataka, India. \\
& $\underline{\text { hebbar.Shahikala@gmail.com }}$ & $\underline{\text { Kavitha_Prasanna@ yahoo.com }}$ \\
\hline
\end{tabular}

\section{ABSTRACT}

Delay tolerant networks (DTNs) have characteristic features such as high end-to end latency, frequent disconnection due to lack of connectivity between the nodes, and opportunistic communication over unsecure wireless links which are not reliable. A challenge is to build a trust management protocol in DTN networks to exhibit a wide range of quality-of-service (QOS) trust such as connectivity and also social trust properties to evaluate other nodes encountered. DTNs are vulnerable to many security threats and hence considered as critical system. In particular, the use of open networks to transmit data offers unprecedented opportunities for security attacks, and allows attackers to compromise information integrity, authenticity, user privacy and system performance. For example, in DTNs, malicious routers can arbitrarily insert false information into the messages. If innocent routers further follow these modified messages from malicious attacker, it generates large amounts of unwanted traffic to the network. Due to resource-scarcity characteristic of DTNs, the extra traffic may pose a serious threat on the operation of DTNs. Unauthorized access and utilization of DTN resources are another serious concern in terms of DTN security.

Key Terms - Delay tolerant network, secure routing, Quality of service trust, social trust.

\section{INTRODUCTION}

Delay-tolerant networking (DTN) is a network designed to operate effectively over extreme distances for instance space communications. Path from source to destination is not present at any single point in time. In DTN network there subsists selfish nodes, malignant nodes and well comported nodes. Low delivery ratio occurred in wireless network due to lack of connectivity is referred to as to as Delay Tolerant wireless sensor Networks. Disruptions could occur due to lack of well-behaved nodes in the network, loss in radio signals, noise or attacks caused by malicious nodes. DTN kind of networks finds its application in critical systems. Hence, number of malicious nodes, selfish nodes in the network is found abundantly. Selfish nodes are group of nodes which exempt themselves from protocol execution to save energy, leading to which the packet is not forwarded to destination. Malicious nodes are those set of nodes which brings attacks in the system like, holding the packet until time to live expires and later trying to send packets, dropping packets unnecessary leading to traffic in the system. Therefore, care must be taken to avoid packets being given to malicious or selfish nodes. To deal with this episodic connectivity in DTN, store-carry-and- forward of messages through buffer is used for delivery of packets towards the destination. Application domains of DTNs include mobile Wireless Sensor Networks (WSNs) for wildlife tracking, underwater sensor networks, and disaster relief team networks, networks for remote areas or rural areas in developing countries.

\section{ASSOCIATED EXERTION}

The various methods to bring out secure routing in delay tolerant network are described as follows:

\section{EPIDERMIC APPROACH [2]}

Forwarding decision is flooding the entire network with the packet that is needed to be forwarded to the destination.

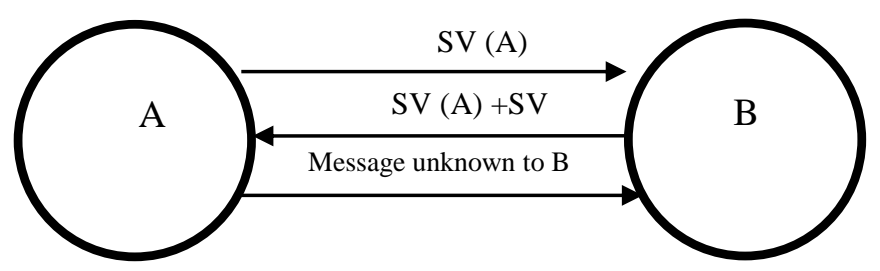

Fig 1: Exchange of summary vector between nodes (SV) which enables the nodes to identify the new message and request them.

Node A creates a copy of the message and sends to node B, if a copy of the packet present in node A is not present in node B. Through Cache maintenance FIFO Queuing perhost; new messages are given preference over old ones in terms of buffer availability. Since the messages floods the entire network, redundant copies are formed which leads to multiple identities. A possible attack against the system has been identified as Sybil attack.

\section{SPRAY AND WAIT [7]}

To overcome quite a few drawbacks associated with epidemic, spray and wait came into picture which puts an upper bound on number of copies of packet that can be generated along with the message to be sent to the destination. This method is consists of two phases namely Spray phase and Wait phase. In spray phase source node simply forwards the message or data packet to the intermediate node. The source of a message initially starts 
with L copies; any node A that has $\mathrm{n}>1$ message copies, and confrontation another node $\mathrm{B}$ with no copies, hands over to $B, n / 2$ and keeps $n / 2$ for itself; when it is left with only one copy, it switches to direct transmission.In wait phase if destination is found it will perform direct transmission. It means node itself forward the packet to the destination. To improve the performance of spray and wait, binary spray and wait scheme is used. After this source node perform the direct Transmission.it produces fewer transmission of packets when compared to epidemic routing. However, the technique works better when all nodes in the network are identical distributed with the same probability distribution with minimum number of nodes.

\section{BAYSIAN SYSTEM [4]}

In this system instead of just flooding the packet blindly in network a thought based on knowledge of previous encounters about the network is considered to route packet. The framework has been developed using Bayesian formulation specifically beta reputation system, recommendation of nodes representation, updates and integration. Once the packet is passed to other nodes, each node ensures that the packet reaches the destination and formulates the trust value based on reputation scheme. Routing framework involves two phases, classification and packet forwarding. Two attributes are considered in Bayesian routing one is time slot and other is location. Location attribute is considered because in most DTN, the packet delivery probability depends on the physical location of the nodes. Time slot attribute is considered because it gives the time instance of the packet being forwarded.The goal of the first phase is to create an abstract grouping of nodes based on certain prior information about the network. Gradient routing is applied in the next phase, where a node tries to forward the packet to a neighbour who has higher affinity with the destination, in this process the packet moves towards destination. The affinity of a node with the destination is computed as a posterior probability using Bayesian inference and is based on the history of past packet forwarding behaviour which is conditioned on network specific factors such as node location, time etc.Node $\mathrm{i}$ believes that node $\mathrm{j}$ misbehaves(deviates) with probability $\theta$. Beta function is probability distribution of $\theta$.Bayesian classifier based routing decision framework which simplifies the integration of various routing attributes and utilizes the repetitive nature of people centric DTN in making better routing decisions. Trust evaluation is based only on node's QOS property (connectivity) and flat wireless sensor network architecture which may not be scalable.

4. PROBABILISTIC ROUTING PROTOCOL USING HISTORY OF ENCOUNTERS AND TRANSITIVITY PROTOCOL (PROPHET) [3]

The Probabilistic Routing Protocol using History of Encounters and Transitivity algorithm is one of the most prominently used routing algorithms for DTN routing. To communicate through these kinds of networks, PROPHET uses the history of nodes encounter. The basic idea is that if a node has encountered destination in many iteration has greater probability to encounter the destination again. Transitivity technique is used to determine the next hop for a node. However, since the method is based on only probability doesn't work well in many scenarios.

\section{FUZZY TRUST MODEL [8]}

Fuzzy system determines the routing path for packets when energy level of a node along with probability of delivery of packets is given as input. It resembles human decision making as it generates a higher knowledge of the next message carrier in the network. Messages are flooded with limited Time-To-Live (TTL), and delivered to the destination when it finds a node which has greater number of encounters towards the destination. It focuses on only probability of delivery and energy value of nodes in the network.

\section{GAME THEORY [5]}

A game between sensor nodes and three factors namely cooperation, reputation and quality of security. Cooperation between nodes means there is more reliable data communication between nodes, by merging these factors the trust value is calculated. In this method there is a high detection rate of detecting selfish nodes with less energy usage. However the method turns out complex as iterations passes, which makes it hard to implement.

\section{ORACLE BASED ROUTING [10]}

Knowledge centers (Oracles) are used to make routing decisions. These Oracles possess all information of future movements, and computes the best set of forwarding decisions. This technique is useful to compare against various existing schema. This type of routing algorithms takes an advantage of predictable information about network topology. Based on this information they compute the route. The information based on traffic demand, schedule of contacts and queuing in forwarding process are used in complete knowledge protocols.

This method depends only on the efficiency of the algorithm that is used to calculate the best route and the type of knowledge oracles used in determining the best route towards the destination.

\section{DYNAMIC TRUST MANAGEMENT TECHNIQUE [11]}

Various previous methods have focused on factors such as either connectivity pattern of a node or energy levels of a node or it could be behaviour of nodes. However, merging 
two or more factors will bring out a secure routing and also maximizing the application performance. The framework is depicted in the following figure 4.

1. Social Trust Calculator - Trust based on behaviour (healthiness and selfishness) of nodes is calculated.

2. QOS Index Calculator - Trust based on energy levels of a node and connectivity pattern of a node towards the destination is calculated.

3. Trust-Security Integrator - The trust value and security index is converted into a combined index in this module.

4. Update module - whenever new nodes are added to the network and when there is a change in number of malicious nodes the protocol gets executed.

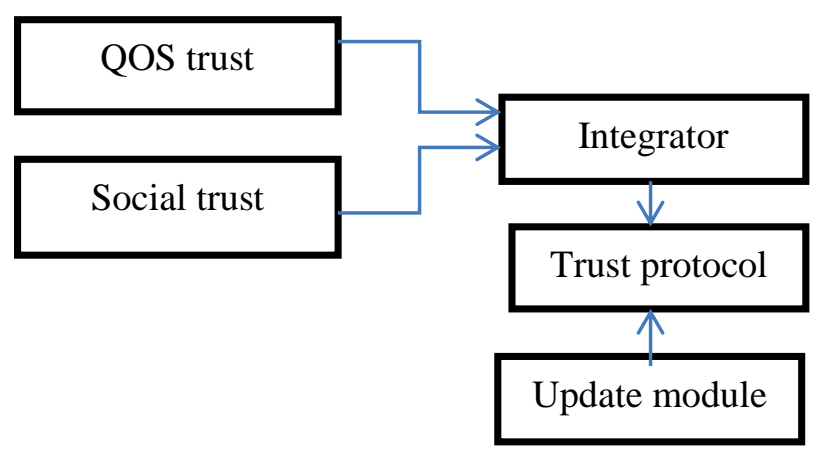

Fig 4: DTN approach which involves QOS trust and social trust and combining both factors

In DTN routing, message delivery ratio and message delay are two important factors. Message delivery ratio includes healthiness, unselfishness and energy factors of a node is considered to maximize message delivery .On the other hand, Message delay includes connectivity of a node towards destination is considered to minimize the delay. Delay tolerant networks are often encountered in military network environments where end-to-end connectivity is not guaranteed due to frequent disconnection or delay. Combining two or more factors of a node such as connectivity, energy, behaviour of nodes (healthiness \& unselfishness) to select the next message carrier towards the destination maximizes application performance. To develop packets or messages which contain all the data needed to forward which is based on information-centric networks (ICN).Implementing the trust protocol on DTN network are some of the future works associated with this technique.

\section{CONCLUSION}

Security is major criteria in any network communication. Various methods which perform secure routing on DTNs have looked into factors such as either the connectivity capability of a node towards the destination or energy levels of a node(QOS) to perform basic routing or it could be only behaviour of nodes in network such as healthiness and unselfishness(social trust). By considering connectivity factor message delay is reduced and by ensuring that the packet is not forwarded to the nodes that deviate from normal behaviour for instance malicious or selfish nodes the message delivery ratio is reduced. However, with an idea of merging two or more factors will bring out a better secure routing.

\section{REFERECES}

[1] M. K. Denko, T. Sun, and I. Woungang, "Trust Management in Ubiquitous Computing: A Bayesian Approach," Computer Communications, vol. 34, no. 3, 2011, pp. 398-406.

[2] A.Vahdat and D.Becker. "Epidemic routing for partially connected ad hoc networks". Technical Report CS-2000-06, Department of Computer Science, Duke University, April 2000.

[3]. A. Lindgren, A. Doria, and O. Schelen."Probabilistic Routing in Intermittently Connected Networks". In proc. of the 2003 ACM Mobile adHoc.

4. Ramanathan, R., Hansen, R., Basu, P., Rosales-Hain, R., \& Krishnan, R. (2007). "Prioritized epidemic routing for opportunistic networks". InProceedings of the 1st international mobisys workshop on mobile opportunistic networking. Puerto Rico.

[5] Afrand, "A game theory based approach for security in wireless sensor network", IEEE International conference on Performance, Computing and communication 2005 p 259263.

[6] S. Jain, K. Fall, and R. Patra. "Routing in a delay tolerant network"In Proc. ACM/ Sigcomm, Aug. 2004.

[7] T. Spyropoulos, K. Psounis and C. S. Raghavendra, "Spray and Wait: An Efficient Routing Scheme for Intermittently Connected Mobile Networks," In: ACM SIGCOMM Workshop on Delay-Tolerant Networking, 2005, pp. 252-259.

[8] JunhaiLuo "A trust model based on fuzzy recommendations for MANET," Computer Networks vol 53 2009p 2396-2407.

[9] I. R. Chen, F. Bao, M. Chang, and J. H. Cho, "Supplemental Material for 'Dynamic Trust Management for Delay Tolerant Networks and Its Application to Secure Routing'," IEEE Transactions on Parallel and Distributed Systems, 2013.

[10] Routing Protocols in Delay Tolerant Networks: A Comparative Survey, 2008. S. Ali, J. Qadir, and A. Baig. "Routing protocols in delay tolerant networks - a survey". In Emerging Technologies (ICET), 6th International Conference on, pages 70-75, 2010.

[11] Ing-Ray chen,FenyeBao,Moon Jeong chang,and JinHee cho"Dynamic Trust Mangement for Delay Tolerant Networks and its applications to secure routing"IEEE transactions on parallel and distributed systems, VOL.25,no.5,May 2014 